\title{
Research on aircraft components assembly tolerance design and simulation technology
}

\author{
Wei Wang ${ }^{1, a}$ HongJun $\mathrm{Yu}^{1, b} \quad$ Ahmed Shafeeu ${ }^{1}$ TianHui Gu${ }^{1}$ \\ ${ }^{1}$ Shenyang Aerospace University ,LiaoNing, China \\ awwsh595@163.com, byhj850303@163.com
}

\begin{abstract}
Keywords: Aircraft assembly, Tolerance design, Simulated analysis
Abstract. With the rapid development of aircraft industry, manufacturing department needs to put forward higher requirements for the accuracy of aircraft assembly. It not only affects the overall quality of the aircraft but it also affects the cost of aircraft manufacturing. This paper introduces the ways about the improvement of the assembly accuracy and guarantee of the quality of assembly, based on the conventional tolerance design, using 3DCS tolerance analysis software. It also elaborates on the tolerance design modeling technology and shows the simulation model of how tolerance analysis is set up. The VSA and 3DCS software are compared and the application methods that could be used in the design of aircraft assembly of the 3DCS tolerance simulation analysis software are discussed. The simulation of how the tolerance design is carried out for the assembly of aft fuselage and hatch door.The results are gained about the analysis of the assembly tolerance accordingly with the distribution, it directly analyses the aft hatch door dimensions under coordination of assembling. The necessary improvement and comprehensive optimization about dimensions ensure the quality and efficiency of the assembly of aft aircraft hatch door.
\end{abstract}

\section{Introduction}

In aircraft tolerance analysis, the modeling is extremely important. For the tolerance analysis ,distribution, synthesis, evaluation and inspection are based on the previous model. There are no systematic, complete or quick ways to express these aspects of tolerance for a unified model during the aircraft manufacturing tolerance expression, among the mathematical models of all dependent products. Some show tolerance range change only through the transformation of $3 \mathrm{D}$ model into $2 \mathrm{D}$ model, improper tolerance design Interco-ordination dimension chains are expressed by means of calculation,others show the tolerance variations only in the three dimension.

There are some similarities between the different representation models which local and foreign researchers have come up with. In CAM-I system based on B-rep, Johnson put forward EDT model according to problems posing to the tolerance of entire model. But the EDT model only supports the B-rep solid modeling methods. Requicha developed a type of expression model using V-graph data structure in PADL-2 which is based on CSG.Tolerance information acted as a property of features and attribute layer to make the presentation. B-rep representation uses adjacent surfaces, Surfaces are defined as entity of the object, relationship between faces of the surface act as the fundamental relationship between the volume space. In this model, representation element reaches the tolerance standard form definition. All the tolerance information such as tolerance types, sizes, reach on the referenced standard the form of properties it provides. Through the defined kind of method referring to these properties add the tolerance in CAD system. Express the Internal part size tolerance information and inter part assembly relationship information using shape network model.

\section{Tolerance modeling technique}

According to Studies on tolerance model of various products locally and abroad, expression of size, overall tolerance information quickly is not enough. Especially in terms of tolerance model's unified representation, a perfect model is yet to be formed. In conventional aircraft manufacturing, within tolerance design theory research aspect, the majority remain in the expression of two-dimensional 
drawings. Usually in reference to the two-dimensional drawings, the three-dimensional geometric model is used to calculate and express tolerance methods. In aircraft manufacturing the impact tolerance design has on the assembly cannot be pointed out directly. Currently 3D does not have process information of the products thus cannot have effective support for later work of the assembly tolerance design. From the stage of 3D application situation, we see construction tolerance analysis of the prevailing conditions model shown in Figure 1.

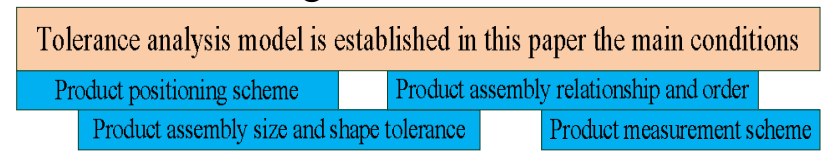

Fig.1 Assembly tolerance analysis model of main conditions

\section{Assembly tolerance design and simulation technique of aircraft components}

\section{Analysis of assembly tolerance design and simulation technique}

Aircraft assembly simulation is a new technology used in the manufacture of modern aircrafts. One could visually simulate the methods on the computer, it resolves product feasibility, Accessibility, verify the reasonableness of the assembly process design and so on. Since Aircraft product assembly is a complex process, high accuracy requirements are laid out for the assembly. In aircraft manufacturing, a reasonable assembly tolerance design ensures the improvement of the assembly accuracy, improving the assembly design efficiency and reduces the manufacture cost, also increases the success rate of the assembly. Aircraft assembly tolerance analysis technology has helped in improving the aircraft assembly quality and efficiency. It has also become the key technology to shorten the manufacturing cycle. Commonly used tolerance design software these days include, eM-Quality, CETOL 6sigma etc. So as to ensure the accuracy in aircraft assembly, during the assembly simulation analysis, use a complete combination of simulation and tolerance analysis software simulation imperative. Assembly simulation software usually DELMIA, 3DCS with VSA and related software. For the assembly tolerance simulation, preparation of Tolerance analysis must be done in the previous simulation model. Most of the current assembly simulation software, during simulation verification, use ideal models without tolerances. Also they don't have any assembly tolerance analysis capabilities. In the aircraft assembly process performance verification procedure, assembly simulation and assembly tolerance analysis are two interrelated aspects. Using the assembly simulation software, after conducting assembly process simulation on the assembly model, then we go to the tolerance analysis calculation. Since the two software are mutually independent, 3D assembly data information may be lost between two different software, or it might be the same software of different versions. This will require a lot of human input values in the model tolerance analysis software, such as fitting assembly sequence, the constituent element of each dimension ring, tolerance cumulative directions and other information. At the same time due to differences in different people's understanding of tolerance information and method of operation, it might lead to a deviation in the analysis results.

\section{Aircraft assembly tolerance design simulation analysis technology based on VSA}

Three dimensional tolerance simulation analysis (VSA) technology which uses simulation of aircraft components assembly process to predict the size, quality and error contribution factor of the product. In the aircraft product design and development phase, it helps the product and process designers analyze and evaluate the parts. Process and tooling doesn't meet the target design. For the determination of components' key feature simulation analysis, the size design requirements cannot be met. The possible design rectifications will be shown through component assembly simulation analysis results. This is a good improvement to make up for the severe tolerance overlap calculations caused by traditional manual complex calculations. It can not consider insufficiency of inaccuracies and other shortcomings of 3D factors and the calculated result. Effectively sustained the design side, manufacture and assembly concepts in early design and development applications of aircraft. Process of VSA application in the aircraft components assembly tolerance design simulation is shown below in figure 2 . 


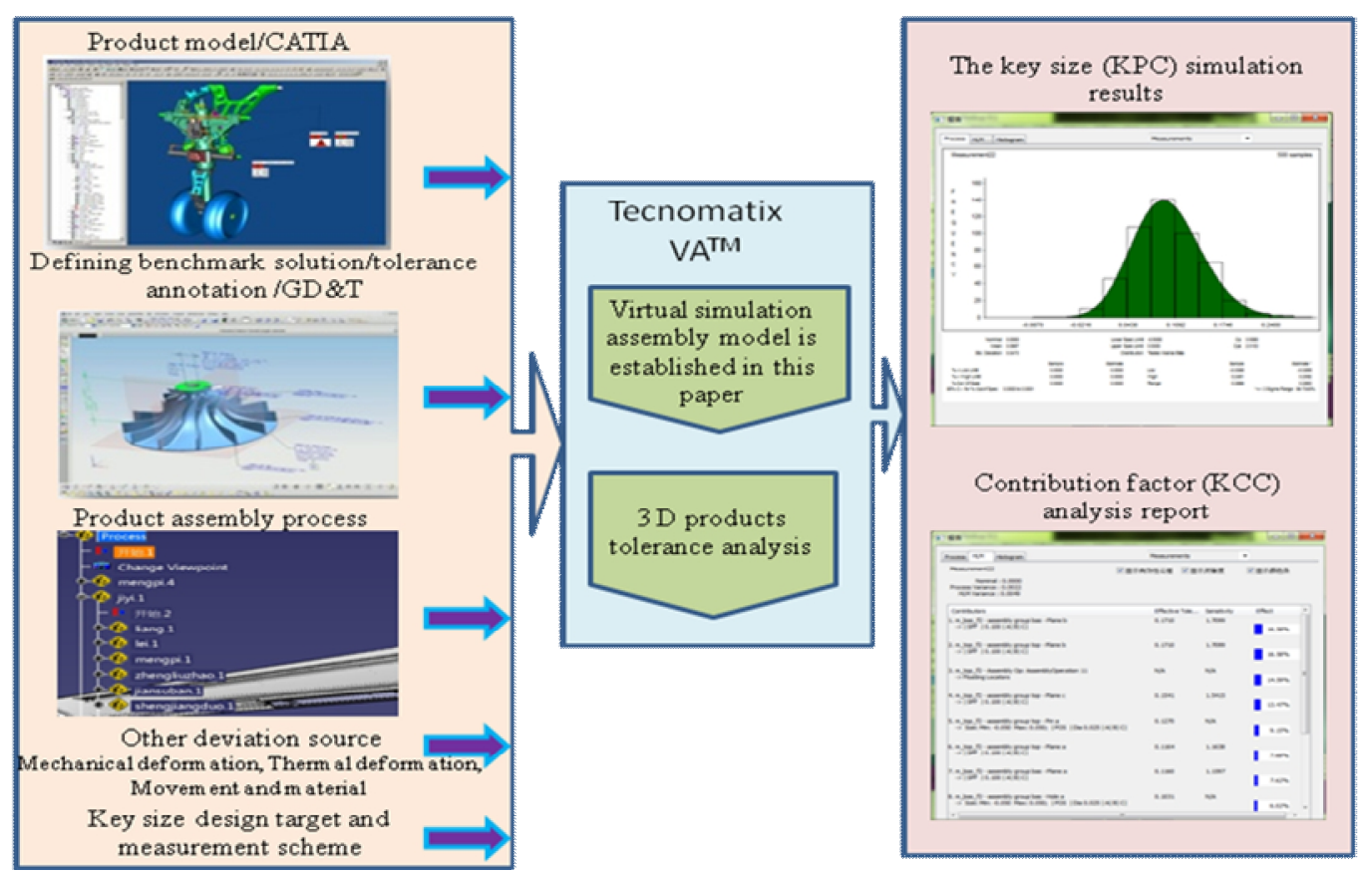

Fig.2 VSA tolerance design process simulation analysis

The 3D design of the aircraft product in the previous format is converted to the needed format for VSA, to import into the software. Check the aircraft components design, datum reference plan and tolerance values/GD\&T diagram. According to the assembly process and procedure diagram set target for simulation analysis of critical dimensions and test plan. At the same time take into account other error factors, such as mechanical deformation and thermal deformation etc. In the simulation analysis VSA software builds a virtual assembly model, in accordance with assembly sequence setting up the tolerances of key locations. After completion of 3D model's tolerance simulation analysis, using the analysis results we could get the size of the error. To determine compliance with design requirements, obtain report of the contribution factors to the errors, in turn to obtain reasonable design of aircraft components and assemblies.

Using the GD\&T, standard, measure points associated with tagging and other information of the input aircraft components, make the 3D tolerance simulation analysis. Through prediction of each part's key features, such as, holes, surfaces, and lines etc., estimate the effect parts will have on the subassembly by dimension chain analysis. By seeing the deviation in error we could identify the source of error. Conjoin assembly operation, is not completely constrained or too constrained as there are many simulation calculations of positioning and installation constraints. Establishment of a unified tolerance library (Tolerance Library), according to different corporate standards, could let different aircraft manufacturers, to share resources, also model can be updated quickly automatically via a shared platform. VSA builds the model through digitized simulation of parameters. Convenient in early simulation analysis, which helps in the prevention of product design problems in order to reduce the manufacturing department's alterations. To achieve the maximum out of the tolerance analysis, also integration with PLM systems, to make collaborative digital prototypes, share resources, business processes, best practices and knowledge accumulation etc. Advantages of using VSA software in aircraft components assembly tolerance design; improvement of design of aircraft in developmental stage, reduce the alterations to the design by manufacturing department, optimize the part assembly process.

\section{Aircraft assembly tolerance design simulation based on 3DCS}

When using 3DCS tolerance analysis software integrated in CATIA V5 environment, there is no need to convert the data model directly to product while importing to CATIA. By analyzing the product components, complete the extraction and manage the component data. Simulate the assembly motion based on Monte carlo method with dimensional tolerance analysis, different ways of measurement simulation during assembly, the final result obtained contains error contribution factors 
and generates a report based on the error analysis. The specific application of 3DCS tolerance analysis software in aircraft assembly process is shown in figure 3.

3DCS tolerance analysis software integrated in CATIA, begins product's 3D design of digital model, aircraft assembly process, product quality reference data and technical requirement and other preparations. When the digital model is put into 3DCS, it will define the part's DCS point, after that the DCS point of the part will be copied into the tooling. Define position locator's diameter tolerance, after finishing the establishment of measurement points, put the product part onto the tooling assembly. Check offset between the locator and its hole. After shifting the offset, determine the tolerance of positioning block surface, and the tolerance of jig orientation and locating pin's position. When a welded junction comes up, define the welded point's DCS point on the part's welded position. The same process applies when copying the part's welded point onto the jig's welded point, define the movement, and make the relevant modifications to the welded part's position tolerance. Define all the needed measurement points and complete the organization of points. Finish the assembly optimization simulation, generate analysis of contribution factors and measurement analysis report. Refer the measurement report to the aircraft component manufacture tolerance design and optimize the assembly sequence of components.

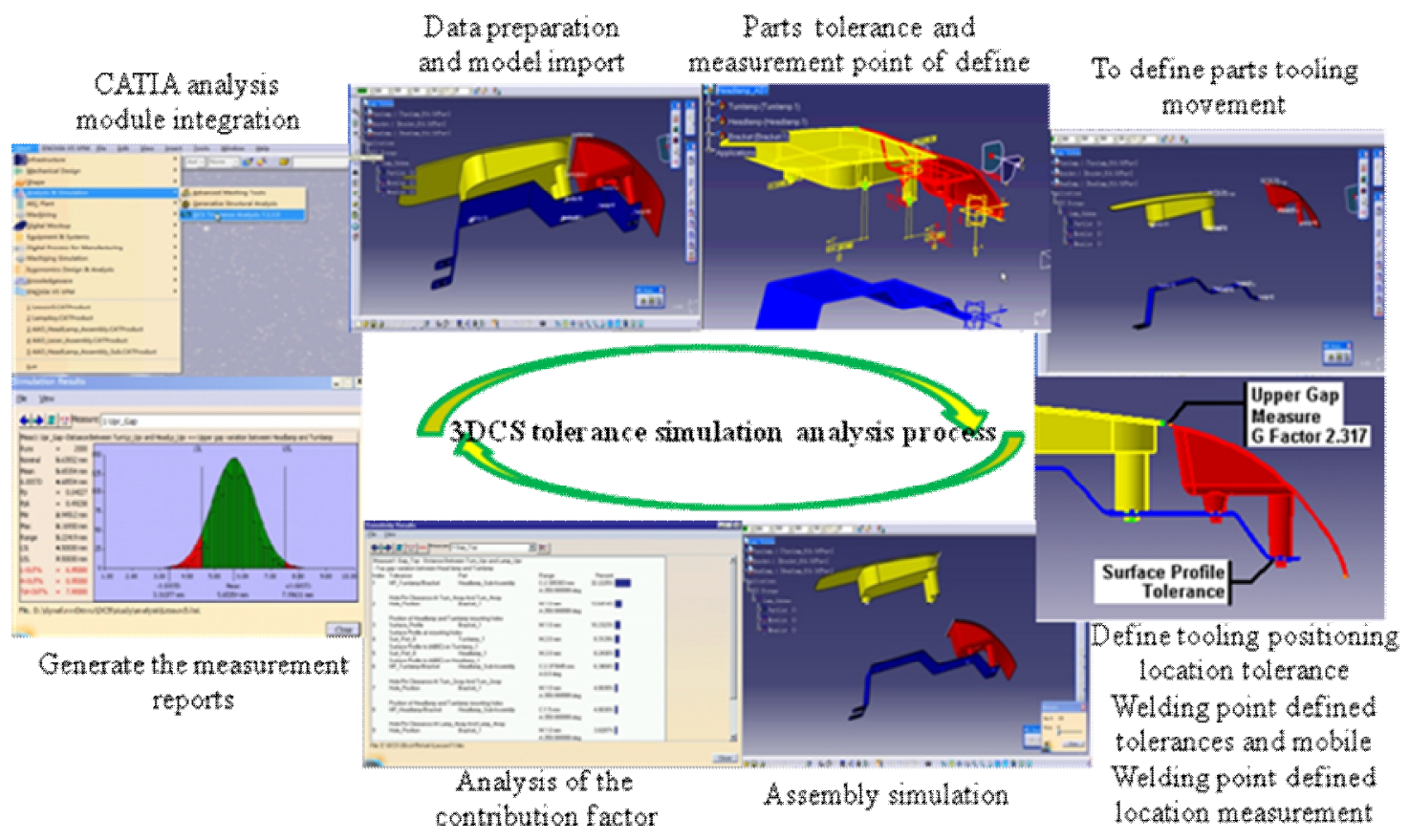

Fig.3 3DCS tolerance simulation analysis process

In 3DCS tolerance analysis software, the part size change can be passed from one level of the assembly to another, also the shape of the parts has a direct impact on the change in level. During size change overlay analysis, factors such as clearance between the parts, slip planes and mating relations, were also taken into account. Before freezing the part design, set up an error analysis model; which could optimize the geometric model also could use points and vectors to show the geometric model. Tolerance of parts have a significant impact on formulating the part manufacture process plan. Within the allowed tolerance user can select the most cost effective process plan. If the tolerance design did not consider the processing cost, we could add the tolerance directly, at the same time by using FT\&A a module automatically in CATIA, the tolerance information can be set up. The product's size change has a big impact on the final assembly process. In the software, Monte carlo method is the most commonly used for tolerance analysis. According to the actual distribution of each dimension, use a random number generator to generate a relevant random number. According to tolerance design's function calculate the value of the closed loop data. Finally according to the distribution of the size of a closed loop, find the corresponding tolerances. The average size of a closed loop $X_{a v}$ :

$$
\mathrm{X}_{\mathrm{av}}=\sum_{\mathrm{i}=1}^{\mathrm{n}} \mathrm{X}_{\mathrm{i}} / n
$$


Closed loop size of extremes $X_{0 \max }$ and $X_{0 \min }, X_{0 \max }=X_{\max }, X_{0 \min }=X_{\min } \quad$, Closed loop upper and lower deviation :

$$
E S_{0}=X_{0 \max }-X_{a v}, E I_{0}=X_{a v}-X_{0 \min }
$$

During the analysis, the assembly sequence of the part, use of fixture as well as form of fasteners have an effect on the analysis results. According to MOVE of the set up assemble the parts, these moves control relationship between the positions of the part in space at different places. In 3DCS, the dimension requirements of aircraft assembly expressed using measurements can be modified, note that target change leads to change in the design, thus changing the product requirements, also could do rework or discard the defective parts to complete. Each part is assigned an error value, and these error assigned parts are assembled according to the assembly plan. Compare the expected output results and simulation output results, at the same time could compare to simulation results in a different dimension management plan. The tolerance of the aircraft parts are according to specified levels, producing tolerance values of different accuracies, which will result in different output results in the analysis. Finding the most problematic areas are quite easy. Advantages of Using 3DCS tolerance analysis technique in aircraft assembly are; it reduces the cost of aircraft part production, improves quality of aircraft parts, reduces aircraft assembly cost, accelerates the assembly process, reducing the aircraft manufacture cycle time.

\section{An aircraft assembly tolerance simulation technology}

Aircraft assembly simulation tolerance analysis combinate simulation and tolerance analysis software to effectively figure out the coordination relationship between good simulation models and actual production dimension. Used 3DCS software to do aircraft aft fuselage and hatch door joint assembly tolerance design simulation. The aircraft aft fuselage and hatch door joint assembly tolerance analysis process is shown in figure 4.

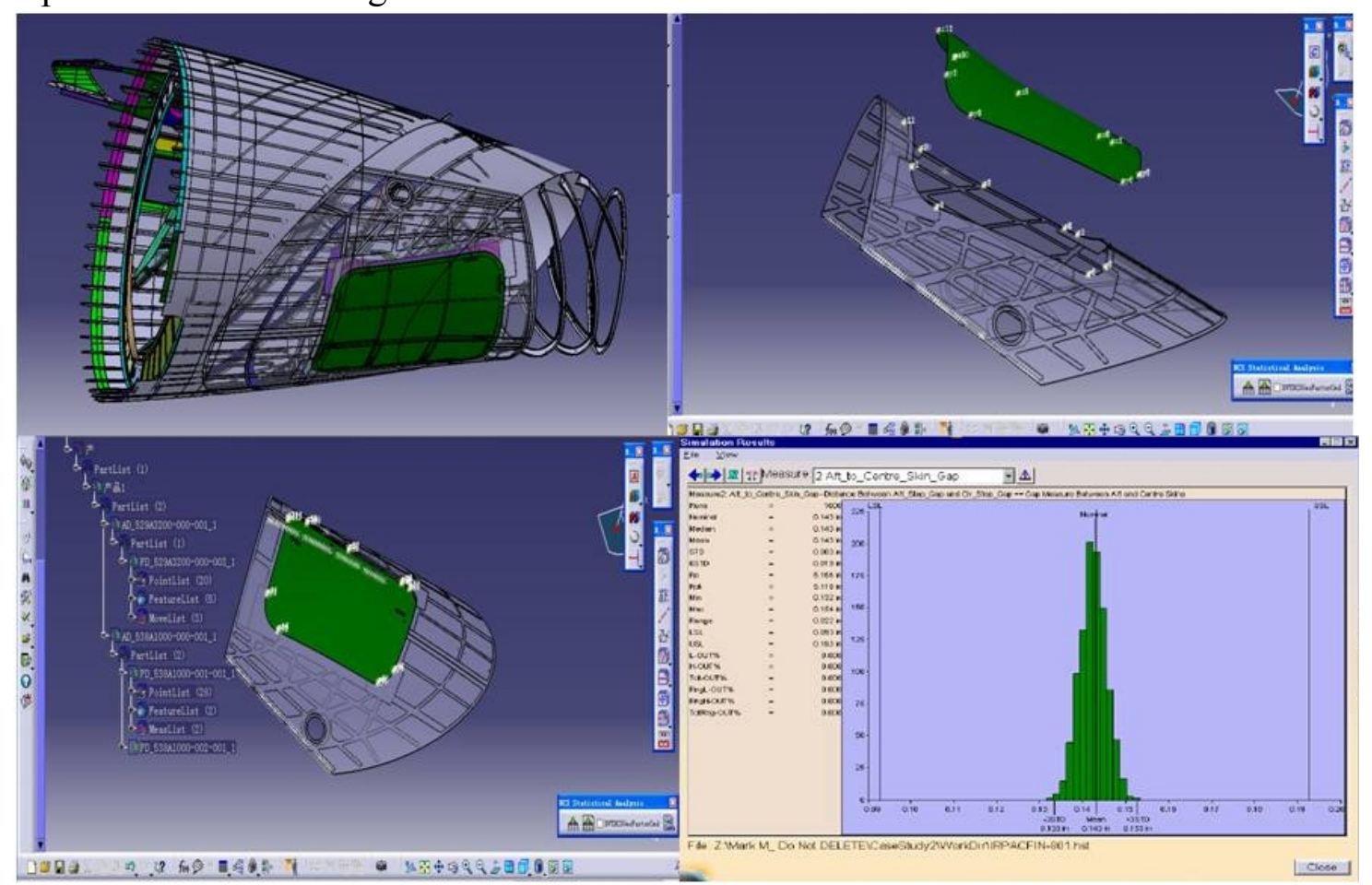

Fig.4 An aircraft aft fuselage and hatch door joint assembly tolerance analysis

During the aft fuselage and hatch door joint assembling process, conducted an effective tolerance analysis on the part of the door and fuselage skin part assembly location. Before the output of simulation results, it must be in accordance with the assembly simulation and tolerance analysis software analysis function. Import the 3D joint assembly of the product in to the software, delete the 
parts which does not need to be analyzed, add the dimension tolerances of the necessary parts of the hatch door and fuselage skin. Define the method for assembling the parts clearly, in order to successfully assemble aft fuselage and hatch door, process margin and assembly clearance are needed. For the clearance between the two parts build DCS points adjusting the measurement between points. Adjust assembly simulation movement, establish key feature points assembly alignment. Finally run it to give the simulation results of the tolerance design. The Contribution factor analysis results from operation results are shown in figure 5.

\begin{tabular}{|c|c|c|c|c|}
\hline \multicolumn{5}{|l|}{ ity Results } \\
\hline \multicolumn{2}{|c|}{ 迶 } & \multicolumn{2}{|c|}{ 氖 } & \\
\hline \multicolumn{5}{|c|}{ 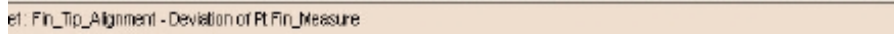 } \\
\hline Foleance & Pairt & Fart & Fange & Percent \\
\hline in_are_loc_tal & $\mathrm{F} \cap 2 \mathrm{~F} 4$ & FPFAN-D01_1 1 & N.0005n $n$ & $24.97 \%$ \\
\hline R4_Fin_Cic_Loo_tal & $\mathrm{F} 4_{-} \mathrm{Fin} 2$ & FPFR-004_1 & MN:0005in & $24.97 \%$ \\
\hline Red_Fn_Crc_loc_ta & $\mathrm{Fr}_{4} \mathrm{Fm}$ & FPFT:-004_1 & noopsin & $22.55 \%$ \\
\hline Gro_Lo_Tol & Fin1_FRt & FPFIN-001_1 & N1:0005n $n$ & $22.55 \%$ \\
\hline 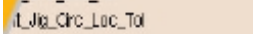 & FRe_4 4xay & Ant_lid_L 1 & N.0005n & $1.16 \%$ \\
\hline Att_liz_ac_loc_ta & Fri_-2way & Ant_lib_1 & $\times 0005 n$ & $0.63 \%$ \\
\hline :R4_Tertiary__Clos__Cro_Loc_Tol & FR4_2way & FPFR:004_1 & No000 $\mathrm{n}$ & $0.63 \%$ \\
\hline RR_fin_lines_Loc_Td & $\mathrm{F}_{i} 4 \mathrm{~F} \mathrm{n}$ & FPPR- 004 1 & N.0005 in & $0.00 \%$ \\
\hline in_Lres_Loc_Tol & Fin1_FR4 & FPFIN-001_1 & M:0005 $n$ & $0.60 \%$ \\
\hline in_Liea_Lac_Td & Fin $2 F_{4}$ & FPPาN-001_1 & M. $0005 \mathrm{n}$ & $0.59 \%$ \\
\hline \multicolumn{5}{|c|}{$\sin$ of res 144 contikutas $=0.750000 \%$} \\
\hline
\end{tabular}

Fig.5 Deviation contribution factor

In the simulation analysis results, parts with pre-set tolerances are recorded during the operation process. From product assembly sensitivity and error contribution factors it can be analyzed, comparison between aft fuselage and hatch joint assembly expected results and simulation output results. At the same time, compare with simulation results under different tolerance distribution conditions.

\section{Conclusion}

In aircraft components assembly using VSA and 3DCS tolerance simulation analysis software, both have high speed analyzing ability of 3D dimension chains the effects of ring of closed ring, and provide effective solutions for coordination problems of dimensions of parts during assembly . Characteristics of 3DCS tolerance simulation technique on aft fuselage hatch door sub assembly design; difficult assembly, difficult measurement analysis, components offer reliable tolerance analysis plan. Also it provided error data source for late hatch and rear fuselage joining assembly. From the aft fuselage and hatch door joining process analysis, it found mating assembly tolerance analysis. Finally it produced information analysis report. It can visually display the error contribution factor, promptly corrected the error, improves accuracy of aircraft assembly, speed up the aircraft development schedule, shortens the manufacturing cycle of aircraft.

\section{References}

[1] Will R. Tolerancing for function[J].CIRP Annals.1988,37(2):1 8

[2] Nassef A.Optimal allocation of types and magnitudes of geometric tolerances[D].Canada: Mcmaster University, 1997

[3] Zhengshu Shen. Tolerance Analysis with EDS/VSA[J]. Journal of computing and information science in engineering.2003,3:95 97

[4] Wayne Cai,Yufeng Long,Ching Hsieh.Variation simulation for digital panel assembly[J].ASME international mechanical engineering congress.2003,5:27 46

[5] Baolin Chen.Theory and algorithm of optimization[M].Beijing:TsinghuaUniversity Press,2004 
